\title{
Potenciar la experiencia y habilidades frente a problemas prácticos en las clases de laboratorio de "Flotabilidad y Estabilidad" Enhancing the experience and abilities in practical problem solving in the lab sessions of the course "Flotabilidad y Estabilidad"
}

\author{
P. E. Merino-Alonso ${ }^{1}$, Javier Calderon-Sanchez ${ }^{2}$, Luis Pérez Rojas ${ }^{2}$ \\ peleazar.merino@upm.es, javier.calderon@upm.es, luis.perezrojas@upm.es \\ ${ }^{1}$ DACSON, M2ASAI-IMEIO-ETSIN-UPM \\ Universidad Politécnica de Madrid \\ Madrid, España \\ ${ }^{2}$ DACSON, CEHINAV-ETSIN-UPM \\ Universidad Politécnica de Madrid \\ Madrid, España
}

"No hay mejor aprendizaje que el de la propia experiencia"

Lev Vygotsky

Resumen- En este trabajo se presentan las medidas adoptadas en las prácticas de la asignatura "Flotabilidad y Estabilidad", del grado de "Arquitectura Naval" de la UPM, para potenciar la capacidad de los alumnos de enfrentarse a problemas prácticos y para entender la casuística que ellos involucran (necesidad de planificación, manejo de herramientas, etc.). En concreto, se describen: a) Una actividad complementaria, introducida en el curso 2019/2020, que involucra la realización de un experimento con un barco real; b) La adaptación de las prácticas a formato no presencial en el curso 2020/2021. Este cambio de formato, consecuencia de la situación de pandemia, tiene en principio un efecto negativo en cuanto al objetivo que se persigue. Para tratar de minimizarlo, se introdujo un nuevo concepto de práctica en la que los alumnos realizaron un experimento casero, devolviendo así el aspecto práctico a la actividad, a pesar de la no presencialidad. En este artículo se evalúa la efectividad de las medidas implementadas en cuanto al cumplimento del objetivo de mejorar la capacidad de los alumnos de enfrentar problemas prácticos, así como de otros objetivos que se buscan con las prácticas. $\mathrm{El}$ artículo se cierra con conclusiones y propuestas de trabajo futuro.

Palabras clave: prácticas de laboratorio, problemas prácticos, planificación de proyectos, trabajo manual, aplicación industrial, covid-19.

Abstract- In this work, we present a set of measures adopted in the course "Flotabilidad y Estabilidad" from Naval Architecture degree at UPM, oriented towards enhancing the capacity of students to deal with practical problems and to understand the issues associated to them. Precisely, we describe: a) A complementary activity introduced in the course 2019/20, where an experiment with a real ship is performed; b) How the practical contents have been adapted to the online format as a consequence of COVID pandemic. Such a change has a negative impact on the objectives that are pursued. In order to minimize it, a novel idea is introduced. This is based on making the students perform their own experiment at home, giving them the opportunity to realize a practical application, despite the online format. In this article, the fulfilment rate of the objectives is evaluated. Finally, conclusions and future work proposals are drafted.

Keywords: lab-sessions, practical problems, project planning, handwork, industrial application, covid-19.

\section{INTRODUCCIÓN}

Un elemento muy importante en la formación del ingeniero es el contacto con problemas reales, prácticos, relacionados con el trabajo manual y las actividades del taller, del laboratorio o del astillero (en caso del ingeniero naval). Es importante que el ingeniero conozca y haya experimentado por sí mismo las dificultades prácticas que entraña el trabajo manual. Ello le permite tener en cuenta todas estas cuestiones a la hora de diseñar. Este rasgo, que consideramos fundamental en un buen profesional, es algo que nosotros, como docentes, buscamos transmitir a los alumnos en el mayor grado posible. En una charla informal con el CEO de un importante astillero en España que fabrica embarcaciones de fibra, éste comentaba, refiriéndose a cierto ingeniero: "Me gusta trabajar con él, porque nunca te diseña un casco para el que no puedas construir un molde simple. Él tiene siempre en cuenta todo eso, como tiene que ser".

La importancia de la propia experiencia en el aprendizaje ha sido señalada por numerosos autores (Bloom, 1956). Este aspecto del aprendizaje es especialmente importante en la formación de profesionales como ingenieros o médicos, que no solo necesitan "saber", sino que necesitan "saber hacer".

Otro elemento fundamental, al que no se le presta demasiada atención en la formación estándar del ingeniero, es la importancia de una planificación cuidadosa a la hora de abordar tareas manuales o de carácter práctico (como actividades industriales o de laboratorio). El fin de esta planificación es observar todas las dificultades posibles que pueden presentarse y anticiparse a ellas. La importancia de una planificación cuidadosa y de la anticipación en proyectos de ingeniería es de sobra conocida entre los industriales y ha sido además puesta de relieve en numerosos estudios, e.g. (Gibson et al., 2006).

Otras habilidades importantes que se busca que el alumno desarrolle mediante las prácticas de laboratorio son: la capacidad de manejar los errores experimentales de manera correcta en los cálculos; la capacidad de realizar un informe detallado, bien presentado, recogiendo el fundamento de la experiencia realizada; la capacidad de anticiparse y lidiar con imprevistos a la hora de la realización de los experimentos, etc. 
Adaptar unas prácticas de laboratorio para lograr una alta efectividad en la adquisición de dichas habilidades es una tarea compleja. En este curso en particular, la situación de pandemia que atraviesa la sociedad ha supuesto una dificultad añadida. A fin de frenar la propagación del virus, muchas actividades presenciales (incluidas las docentes) han sido adaptadas a un formato no presencial. Esto dificulta, por supuesto, la intención de acercar al alumno a un conocimiento práctico, que requiere de la experiencia directa.

En este artículo se presenta el plan aplicado en la ejecución de las prácticas de la asignatura "Flotabilidad y Estabilidad", del grado de Arquitectura Naval de la Universidad Politécnica de Madrid (UPM). En primer lugar, se expondrán los objetivos concretos que se busca con esta actividad docente. A continuación, se presentarán las prácticas tal como se realizaban en años anteriores a la pandemia, así como la actividad extraordinaria "Experiencia de Estabilidad del Barco Solar del Retiro", llevada a cabo como complemento a las prácticas en el curso 2019/2020. Después, se presentará la adaptación llevada a cabo durante el curso 2020/2021, en el que, por prudencia ante la incertidumbre inicial sobre los mecanismos precisos de contagio de la covid-19, se decidió hacer en formato no presencial. Finalmente, se evalúa el impacto de las medidas adoptadas respecto a los objetivos propuestos. El artículo se cierra con conclusiones y propuestas de trabajo futuro.

\section{CONTEXTO}

La asignatura en la que se enmarcan estas prácticas, "Flotabilidad y Estabilidad", trata sobre los fundamentos físicos que explican la estabilidad y la flotabilidad de las embarcaciones. Además, analiza el efecto que tienen sobre dichas características los diferentes parámetros que definen el casco y otros elementos tales como la ubicación de la carga, el estado de la mar, etc. Se trata de la primera (en orden cronológico en la programación docente) de una serie de asignaturas que cubren el programa de lo que denominamos, tal como fue definido por Carlos Godino en (Godino-Gil, 1956), Teoría del Buque. El programa de prácticas para estas asignaturas seguido en la Escuela Técnica Superior de Ingenieros Navales de Madrid fue descrito con detalle en el artículo (Pérez-Rojas, 1996). El planteamiento presentado en el mismo, aprovechando las instalaciones del canal de ensayos hidrodinámicos de que dispone la Escuela, supuso un gran avance pedagógico. En él se introdujeron ideas como el uso del Canal de Ensayos como herramienta para acercar a los alumnos a la realidad del sector; la realización de actividades complementarias como visitas a astilleros, etc.

En este documento se señalan también algunas de las debilidades del planteamiento inicial, entre las que se destaca la poca participación del alumno: "El carácter apuntado en el apartado anterior hace que la participación directa del alumno sea escasa y puede convertirse en una mera práctica de observación. Aunque esta situación siempre encierra algo de positivo, está claro que no es la optima”, (Pérez-Rojas, 1996).

$\mathrm{Si}$ bien en el caso particular de las prácticas de "Flotabilidad y Estabilidad" la participación de los alumnos es mayor en comparación con otras asignaturas de las que componen el bloque de Teoría del Buque, el espíritu de los cambios que se presentan en este trabajo es el de potenciar aún más este aspecto, fomentando así el desarrollo de las capacidades del alumno ante los problemas prácticos y aumentando su experiencia ante los mismos.

\section{A. Prácticas de Flotabilidad y estabilidad en años anteriores}

En el planteamiento de la asignatura se contemplan cinco sesiones de prácticas. Dos de ellas son de carácter introductorio: los alumnos las realizan por grupos fuera del aula. Estas dos sesiones no se contemplan en este artículo. Las otras tres, son de carácter práctico, a realizar por grupos en el laboratorio. Estas tres prácticas, que denominaremos en adelante "prácticas de canal", son:

1. Práctica de canal 1: Experiencia de Estabilidad.

2. Práctica de canal 2: Estabilidad transversal a grandes ángulos.

3. Práctica de canal 3: Efecto del traslado de pesos en la estabilidad transversal.

Todas ellas se realizan de forma presencial en el laboratorio utilizando modelos simplificados como el que se muestra en la Figura 1. En general, se pide a los alumnos que entreguen un informe, único por grupo y práctica, donde contestan una serie de cuestiones que se les plantea, y que involucran cálculos donde se manejan los conceptos clave de la asignatura. En ellos, deben tener en cuenta los errores de medida, las cifras significativas etc. Además de ello, para realizar los cálculos del informe, deben tener en cuenta una serie de cuestiones menores pero de total relevancia a la hora de acometer trabajos manuales como experimentos. Nos referimos, por ejemplo, a: deducir una medida a partir de la geometría y la disposición de los elementos en el experimento; determinar qué medidas son necesarias para deducir la posición del centro de gravedad de un cuerpo; etc. Como cierre del informe, se pide a los alumnos que saquen sus conclusiones sobre el experimento y que reflexionen sobre si los resultados concuerdan o no con la teoría y, si no lo hacen, que expliquen a qué achacan esta discordancia.

\section{B. La experiencia de estabilidad}

La experiencia de estabilidad es uno de los conceptos más importantes de los que tratan las prácticas de canal. Se trata de una técnica que se aplica a barcos reales para conocer la altura de su centro de gravedad. Para su realización se requieren los siguientes elementos:

- Un péndulo para medir los ángulos.

- Un tanque con un líquido (aceite o agua) en el que se sumerge el extremo del péndulo, a fin de amortiguar sus oscilaciones.

- Un conjunto de pesos conocidos ubicados en posiciones determinadas que se mueven de una banda a otra.

Esta técnica es un método experimental de total actualidad y vigencia. Se considera así importante que los alumnos conozcan su fundamento y se familiaricen con ella lo máximo posible, ya que constituye una herramienta fundamental en la realidad del sector naval. 


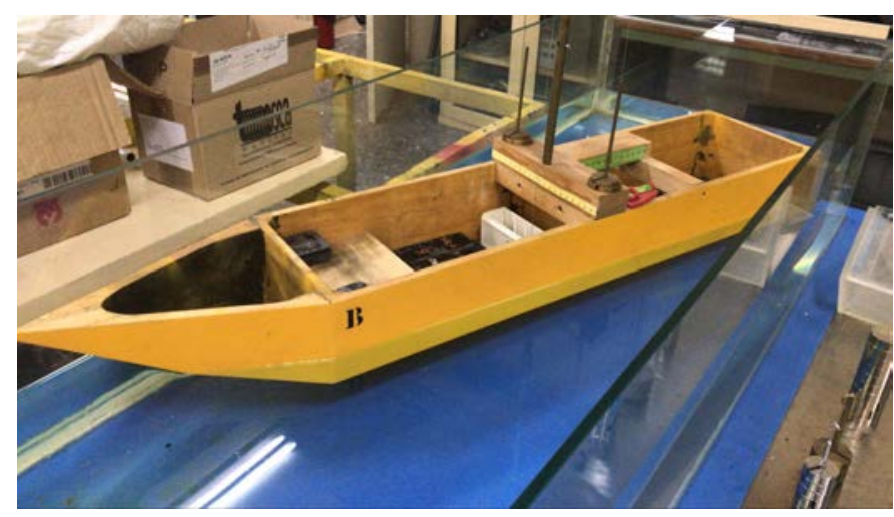

Figura 1 Geosim, modelo utilizado en las prácticas de canal 1 y 2

En la Figura 2 se muestra una imagen de una experiencia de estabilidad practicada a un velero ligero de la clase 420 . La inclinación del mástil da idea de la escora generada por el desplazamiento de pesos.

\section{Habilidades a desarrollar mediante las prácticas de Canal}

Aparte de la revisión de los conceptos de la asignatura Flotabilidad y Estabilidad, las prácticas de Canal se consideran una herramienta fundamental para desarrollar habilidades transversales. Como se ha mencionado antes, la principal de ellas es la habilidad para lidiar con problemas prácticos. Este concepto puede desgranarse en los siguientes aspectos:

1. Comprensión de que, a nivel práctico, siempre se presentan dificultades añadidas que no existen a nivel teórico o conceptual.

2. Comprensión de la importancia de una cuidadosa planificación antes de acometer tareas prácticas.

3. Comprensión de la importancia de tener en cuenta los aspectos constructivos o de ejecución de una cierta tarea a la hora del diseño.

4. Familiarizarse con el manejo de herramientas, dispositivos varios y aparatos de medida.

5. Familiarizarse en general con el trabajo de laboratorio, taller, astillero, etc.

6.

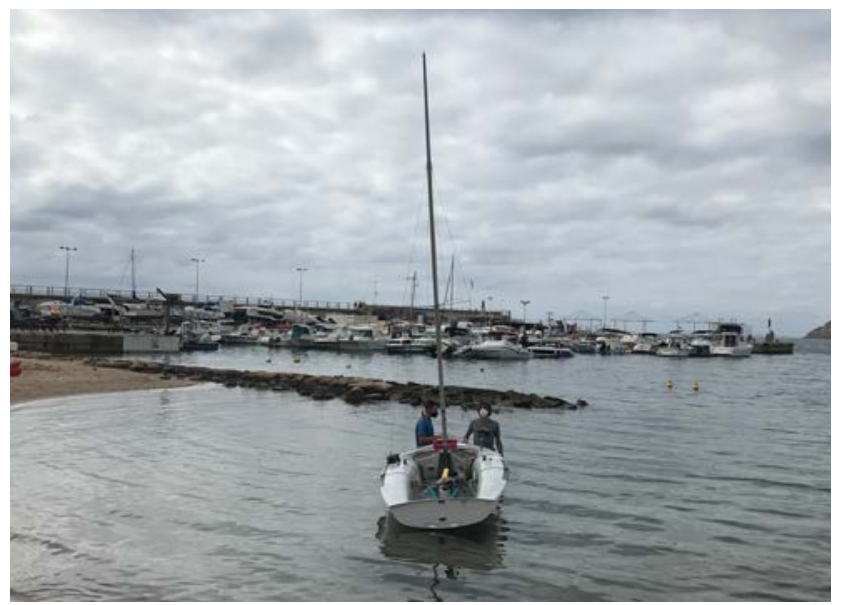

Figura 2 Experiencia de estabilidad con un velero de la clase 420
Las prácticas deben constituir además una ocasión para que los alumnos ejerciten estas capacidades, siendo para muchos el primer contacto con una realidad más cercana al mundo industrial que las clases teóricas.

Además de ello, las prácticas buscan desarrollar también habilidades como:

7. Manejo de errores de medida en los cálculos.

8. Desarrollar la capacidad de juicio de los alumnos hacia los resultados de un experimento, comprendiendo si los resultados son razonables o no, e identificando las fuentes de error.

9. Aprender a plasmar en un informe los resultados de un experimento.

10. Aprender a realizar un informe adecuado, citando correctamente las figuras y tablas, respetando un orden en la exposición, indicando claramente los cálculos de manera que sean reproducibles, etc.

\section{DESCRIPCIÓN}

Pasamos ahora a describir las distintas acciones llevadas a cabo a lo largo de los dos últimos cursos para potenciar los aspectos antes mencionados. En primer lugar, describiremos la experiencia complementaria "Experiencia de Estabilidad del Barco Solar del Retiro", implementada en el curso 2019/2020. A continuación, describimos la adaptación de las prácticas adoptada durante el curso 2020/2021 a formato no presencial. Primero se presenta la adaptación propuesta para la práctica uno, en la que se decidió sustituir la práctica tradicional por la realización de un experimento casero. A continuación se presenta la adaptación de las prácticas 2 y 3, en las que se mantuvo intacto el trabajo de realización de cálculos y elaboración del informe pero se sustituyeron las prácticas por sendos vídeos explicativos.

Las medidas llevadas a cabo para mejorar las prácticas de Flotabilidad y Estabilidad se pueden generalizar, en el contexto de un programa de prácticas de laboratorio de cualquier asignatura de ingeniería, a lo siguiente:

- Incluir un experimento con elementos reales, en vez de modelos, fuera del laboratorio y con el que los alumnos entren en contacto con la realidad del sector.

- Incluir un experimento totalmente casero, fácilmente realizable por los alumnos con materiales cotidianos, que les obligue a realizar una planificación y a enfrentar el proceso de diseño del experimento.

Además, se puede considerar la medida de sustituir una práctica por un vídeo que permita a los alumnos realizar los cálculos aunque no hayan realizado la práctica presencialmente. Es importante aclarar que esta medida se tomó únicamente en respuesta al problema puntual de la pandemia, no como una propuesta de mejora. Aunque consideramos que esta medida empobrece las prácticas como herramienta educativa, puede ser útil en circunstancias extraordinarias como la vivida con la pandemia. 


\section{A. Experiencia de Estabilidad del Barco Solar del Retiro}

En el curso 2019/2020 se propuso a los alumnos de la asignatura una actividad complementaria. Ésta consistía en realizar una experiencia de estabilidad, similar a la propuesta en la práctica de canal 1, con el catamarán del estanque del Retiro denominado "Barco Solar" (Figura 3). Se trata de un barco de pasaje de 12 metros de eslora dedicado al ocio en el estanque. Para ello, los alumnos se desplazaron hasta el Parque del Retiro, y bajo las instrucciones de sus profesores, se distribuyeron en el barco, actuando como pesos móviles, y se procedió a realizar la experiencia de estabilidad y la consiguiente toma de datos. Para ello se designó un alumno encargado de cada tarea: uno para "cantar" las lecturas del péndulo, otro para anotarlas y otros 4 para la medición de calados.

Esta práctica tuvo carácter voluntario y permitió la obtención de 1 crédito ECTS, previa realización de un informe con los cálculos pertinentes. La actividad tuvo buena acogida entre los alumnos (como se constata en la sección de resultados a partir de las encuestas realizadas), asistiendo a ella 36 de 58 alumnos matriculados, un $62 \%$ del total. Con esta experiencia se buscaba acercar a los alumnos a la realidad del sector, volver a insistir en la importancia de este experimento en concreto, y enfrentarlos a una situación práctica real, con dificultades añadidas a las del laboratorio.

\section{B. Adaptación a formato no presencial de la práctica 1}

En el formato propuesto para el curso 2020/2021 la práctica 1 pasó a tener carácter individual. Se facilitó a los alumnos un vídeo donde se les mostraban dos experiencias de estabilidad, una de ellas practicada al modelo de laboratorio (Figura 1), y otra practicada a un velero de la clase 420 (Figura 2). En ambos casos se realiza la experiencia con todo detalle, de forma que los alumnos disponían de dos ejemplos completos para comprender el mecanismo de la experiencia. A continuación se les pedía que construyesen ellos mismos un artefacto flotante, utilizando materiales caseros simples (se sugirió la utilización de tuppers, cajas o botellas cortadas) con elementos análogos a los descritos en la Sección 2B, necesarios para realizar la experiencia de estabilidad. Dichos elementos debían ser incorporados al modelo.
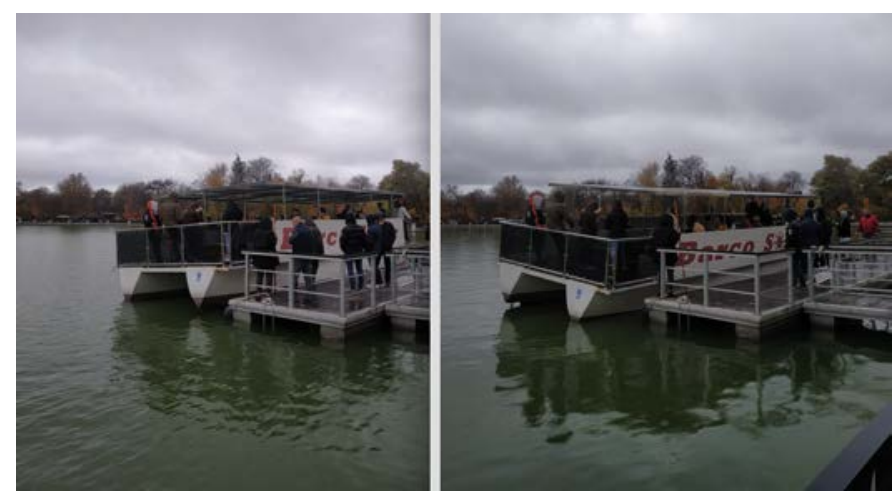

Figura 3 Experiencia de estabilidad del Barco Solar del Retiro, escorando a babor y a estribor

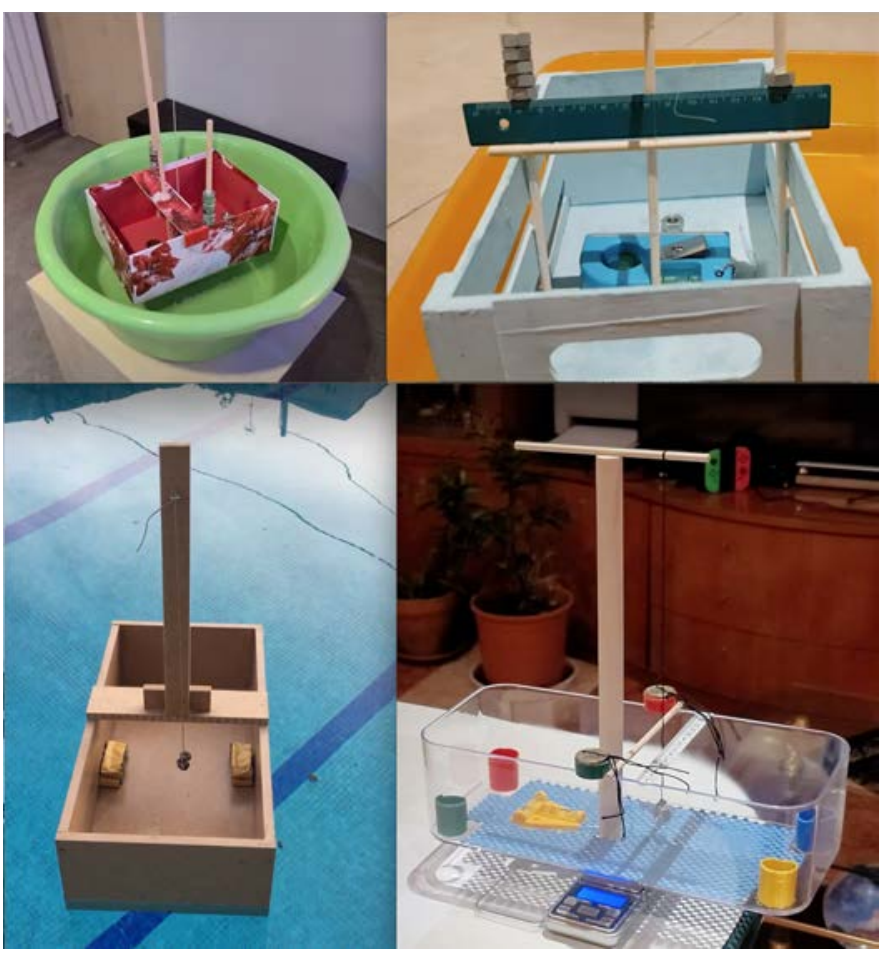

Figura 4 Ejemplos de artefactos realizados por los alumnos del curso 2020/2021 para la práctica 1

Una vez terminado el prototipo, se les pedía que se grabasen en vídeo practicándole una experiencia de estabilidad, de manera que se pudiese después calcular la altura de su centro de gravedad y que entregasen un informe.

\section{Nuevas prácticas 2 y 3: Estabilidad a grandes ángulos y efecto del traslado de pesos}

Para las dos últimas prácticas se propuso un formato similar al de años anteriores, pero sustituyendo la asistencia al canal por unos vídeos explicativos. Los alumnos recibieron un vídeo en el que el profesor realizaba la toma de medidas de la práctica, de la misma forma en que la hubiesen hecho los alumnos en el laboratorio. En los vídeos se daban todos los detalles necesarios para realizar los cálculos, aunque omitiendo explicaciones exhaustivas para que los alumnos realizasen por su cuenta las deducciones necesarias, de forma similar a como hubiesen hecho presencialmente. A continuación se pedía que efectuasen los cálculos de estabilidad, igual que en años anteriores.

Con este formato, se pierde completamente el aspecto de familiarizarse con problemas prácticos, que se busca potenciar en general. No obstante, se decidió utilizar vídeos para las prácticas 2 y 3 por una serie de motivos que exponemos a continuación. En primer lugar, para mantener otros aspectos interesantes de las prácticas: manejo de errores de medida en un caso más estándar; elaboración de un informe adecuado, con toda la casuística de un experimento más complejo; etc. En segundo lugar, la propuesta de la práctica 1, en la que los alumnos debían fabricar un artefacto casero, era una experiencia sin precedentes conocidos en el plan de estudios de la titulación, por lo que se prefirió no convertir las tres 
prácticas a ese formato antes de ver el resultado. Por último, los contenidos que se tratan en estas prácticas son además más difícilmente reproducibles a nivel casero.

\section{Resultados}

Para evaluar el efecto de las novedades introducidas en las prácticas, se han considerado diferentes aspectos. En primer lugar se tendrán en cuenta las notas obtenidas por los alumnos en ambos cursos. En segundo lugar, se ha realizado una encuesta a los alumnos de ambos cursos. Además, en el curso 2020/2021, se ha observado la calidad de los modelos realizados en casa por los alumnos como indicador de su desempeño con las tareas manuales y prácticas.

Las notas de las prácticas de canal obtenidas por los alumnos en los cursos 2019/2020 y 2020/2021 se muestran en la Tabla 1. Las notas son altas en general y han tenido tendencia al alza en el último curso respecto al anterior. Estas notas tienen en cuenta sobre todo el informe de prácticas, por lo que no son significativas de algunos de los aspectos que se buscan potenciar con las medidas presentadas. Además, se quiere destacar que en el último curso se han tenido en cuenta las dificultades añadidas a los alumnos por el hecho de no tener clases teóricas ni prácticas presenciales. En general, se concluye que los resultados académicos de los alumnos en las prácticas de canal son buenos, y han mejorado a pesar de las dificultades del último curso, lo que sugiere que la adaptación que se ha hecho no ha tenido influencia negativa en ese sentido.

\section{Tabla 1}

Medias y desviaciones en las notas, sobre 10, de las tres prácticas de canal en los dos últimos cursos

\begin{tabular}{lcccc}
\hline & \multicolumn{2}{c}{$2019 / 2020$} & \multicolumn{2}{c}{$2020 / 2021$} \\
\hline & Media & Desviación & Media & Desviación \\
\hline Práctica 1 & 6,7 & 1,43 & 8,9 & 1,43 \\
\hline Práctica 2 & 6,89 & 1,7 & 8,12 & 1,78 \\
\hline Práctica 3 & 7,09 & 1,62 & 6,7 & 1,4 \\
\hline Global & 6,89 & 1,59 & 7,91 & 1,54 \\
\hline
\end{tabular}

Por otro lado, se ha realizado una encuesta. Dicha encuesta consta de nueve preguntas en escala de Likert, y una pregunta abierta. La escala se barema de forma que 5 corresponde a "totalmente de acuerdo" y 1 corresponde a "totalmente en desacuerdo". De las nueve primeras, cinco preguntas son comunes y cuatro específicas de cada curso. En las comunes, se les pregunta sobre la utilidad de las prácticas de la asignatura para: a) aprender a enfrentar problemas prácticos; b) aprender a manejar errores experimentales y de medida. En las específicas, se les pregunta por las novedades de cada curso, es decir: la actividad con el Barco Solar del Retiro, y la adaptación de las prácticas a formato no presencial. Las Tablas 2, 3 y 4 muestran los resultados de estas preguntas.

De los resultados de las Tablas 2, 3 y 4 cabe destacar lo siguiente:

- Los alumnos están de acuerdo en que las prácticas de la asignatura ayudan a entrar en contacto con problemas prácticos $(4,29 / 4,47)$.
- Los alumnos no están claramente de acuerdo en que las prácticas ayuden a desarrollar herramientas frente a estos problemas, estando más indecisos a este respecto en el último curso $(4,00 / 3,60)$.

- Los alumnos del curso 2019/2020 consideran en gran medida que la actividad del barco solar es interesante $(4,65)$ y les ayuda a relacionar los conceptos de la asignatura con la realidad del sector $(4,41)$. Piensan que debe mantenerse en cursos futuros $(4,65)$.

- Los alumnos del curso 2020/2021 piensan que se han adaptado bien las prácticas a formato no presencial $(4,13)$, y que el formato más interesante ha sido el de la práctica $1(4,60)$.

- Los alumnos del curso 2020/2021 piensan que la práctica 1 debería mantenerse así en años posteriores $(4,00)$, aunque la desviación en las respuestas es alta en este caso $(1,26)$.

- Los alumnos del curso 2020/2021 no están muy de acuerdo $(3,47)$ en la mayor utilidad del formato propuesto para la práctica 1 frente al tradicional.

\section{Tabla 2}

Resultados de la encuesta, bloque de preguntas comunes. La media y desviación para cada curso (2019/2020 y 2020/2021) se presenta separadas por una barra, "/" y en orden cronológico

\begin{tabular}{lcc}
\hline Pregunta & $\mu$ & $\sigma$ \\
\hline 1. Pienso que las prácticas de la asignatura & $4,29 /$ & $0,57 /$ \\
Flotabilidad y Estabilidad me han servido para & 4,47 & 0,88 \\
entrar en contacto con problemas prácticos, que & & \\
involucran cuestiones esencialmente diferentes a & & \\
los problemas que se plantean a nivel teórico. & & \\
\hline 2. Las prácticas de la asignatura Flotabilidad y & $4,00 /$ & $0,91 /$ \\
Estabilidad me han hecho mejorar mi capacidad & 3,60 & 1,36 \\
para resolver problemas prácticos, que & & \\
involucran la manipulación de herramientas o & & \\
dispositivos (aparatos de medida, etc.). & & \\
\hline 3. Las prácticas de la asignatura Flotabilidad y & $4,59 /$ & $0,60 /$ \\
Estabilidad me han ayudado a comprender que & 4,40 & 0,95 \\
en el trabajo práctico (de laboratorio, industrial, \\
en el astillero, etc.) existen dificultades añadidas \\
distintas de las que aparecen a nivel teórico que \\
hay que tener en cuenta para acometer con éxito \\
este tipo de tareas. \\
\hline $\begin{array}{l}\text { 4. Las prácticas de la asignatura Flotabilidad y } \\
\text { Estabilidad me han servido para entender cómo }\end{array}$ & $3,00 /$ & \\
manejar los errores de medida que se cometen a & & \\
nivel experimental. & & \\
\hline 5. Las prácticas de la asignatura Flotabilidad y & $4,12 /$ & $0,76 /$ \\
Estabilidad me han servido para entender la & 4,13 & 1,15 \\
casuística de la realización de experimentos (la & & \\
necesidad de reflexionar antes de actuar, \\
observar los posibles problemas que puedan \\
presentarse, planificar las actuaciones, etc.). & \\
\hline
\end{tabular}

De las respuestas a la pregunta abierta “¿Qué sugerencias tienes de cara a mejorar las prácticas de la asignatura en cursos futuros?", se han seleccionado las más significativas, por aparición y por relevancia, a juicio de los profesores. De los alumnos del curso 2020/2021, el 35\% señala de alguna forma 
que prefiere la presencialidad y el $14 \%$ señala el formato elegido para la práctica 1 como un acierto. De todas las respuestas contando ambos cursos, casi el $10 \%$ se refieren a que los guiones explicativos son mejorables, incidiendo en que no está bien claro lo que debe contener el informe que se les pide realizar. Es significativo que el $22 \%$ del total señalan que no hay nada mejorable.

\section{Tabla 3}

Resultados de la encuesta, en el bloque de preguntas específicas, para el curso 2019/2020

\begin{tabular}{|c|c|c|}
\hline Pregunta & $\mu$ & $\sigma$ \\
\hline $\begin{array}{l}\text { 6. La actividad "Experiencia de Estabilidad del } \\
\text { Barco Solar del Retiro" es interesante para } \\
\text { afianzar los conocimientos aprendidos en las } \\
\text { clases de teoría y en las prácticas. }\end{array}$ & 4,65 & 0,76 \\
\hline $\begin{array}{l}\text { 7. La actividad "Experiencia de Estabilidad del } \\
\text { Barco Solar del Retiro" me ha ayudado a } \\
\text { relacionar los conceptos estudiados en clases de } \\
\text { teoría y prácticas y la realidad del sector naval. }\end{array}$ & 4,41 & 0,77 \\
\hline $\begin{array}{l}\text { 8. Pienso que la actividad "Experiencia de } \\
\text { Estabilidad del Barco Solar del Retiro" debería } \\
\text { mantenerse en años siguientes. }\end{array}$ & 4,65 & 0,76 \\
\hline $\begin{array}{l}\text { 9. La actividades como la "Experiencia de } \\
\text { Estabilidad del Barco Solar del Retiro" deberían } \\
\text { potenciarse y extenderse a otras asignaturas } \\
\text { siempre que sea posible. }\end{array}$ & 4,65 & 0,76 \\
\hline
\end{tabular}

\section{Tabla 4}

Resultados de la encuesta, en el bloque de preguntas especificas, para el curso 2019/2020

\begin{tabular}{llcc}
\hline Pregunta & $\mu$ & $\sigma$ \\
\hline 6. Pienso que el formato de práctica más & 4,6 & 0,88 \\
interesante ha sido el implementado para la & & \\
práctica 1, en el que tuvimos que reproducir una \\
experiencia de estabilidad en casa, con elementos \\
cotidianos. \\
\hline 7. La práctica 1, tal como se ha planteado este 3,47 & 1,31 \\
curso, es más útil para entrar en contacto con \\
problemas prácticos (como los que pueden \\
aparecer en el trabajo de laboratorio, la industria, \\
astillero, etc.) que las prácticas de laboratorio \\
tradicionales. \\
\hline $\begin{array}{l}\text { 8. Pienso que, en general, se han adaptado bien las } 4,13 \\
\text { prácticas de FyE a formato no presencial. }\end{array}$ \\
\hline 9. Creo que es interesante mantener la práctica 1 \\
en el nuevo formato (fabricación de un artefacto \\
casero y realización de la experiencia de \\
estabilidad con dicho artefacto) de cara a años \\
posteriores.
\end{tabular}

Otro elemento que se utilizará en la evaluación son algunos de los modelos presentados por los alumnos (Fig. 4). Se aprecia cómo los alumnos han tenido que plantear un proceso constructivo con cierta complejidad para elaborar los artefactos, como también ponen de manifiesto algunos de los vídeos enviados. Esto es un elemento en favor de este formato de práctica, que se plantea como una opción interesante para mantener de cara a futuros cursos por el grado de planificación que requiere para abordar la construcción del mismo.

\section{CONCLUSIONES}

De lo expuesto anteriormente se concluye que, en general, las nuevas medidas adoptadas (Experiencia de estabilidad del Barco solar del Retiro, y el formato propuesto para la práctica 1 durante el curso 2020/2021) han servido para acercar a los alumnos a la casuística del trabajo de laboratorio y los problemas prácticos. Puede decirse además que el efecto de la no presencialidad durante el curso de la pandemia se ha visto minimizado por el enfoque planteado, aunque queda claro que los alumnos valoran más las actividades presenciales. A la vista de los resultados de la Tabla 4, se concluye que los alumnos están muy de acuerdo en que el formato más interesante de los dos propuestos en el curso 2020/2021 (vídeos frente a experimento casero) es el de la Práctica 1. No obstante, aunque sí les parece que sea interesante mantener este formato, no parecen tan de acuerdo en que sea preferible a una práctica presencial. Los autores interpretan de ello que los alumnos valoran más las prácticas en el laboratorio, y que el formato propuesto para la práctica 1 , puede mantenerse en esa práctica pero no extenderse al conjunto de todas ellas.

A partir de lo expuesto en el apartado de resultados, se proponen varias acciones para profundizar en la línea de trabajo presentada. En primer lugar, recuperar la actividad del barco solar, que no se llevó a cabo en el curso de la pandemia y afianzarla como complemento a la asignatura. En segundo lugar, se mantendrá la práctica 1 como una actividad a realizar de manera individual por los alumnos, en la que diseñen su propio artefacto casero y le practiquen una experiencia de estabilidad. Ello se complementará con una sesión en el laboratorio, en la que el profesor propondrá a los alumnos un ejemplo de la experiencia completa, incluyendo los cálculos, para que sirva como referencia. En tercer lugar se volverá a formato presencial para las prácticas 2 y 3 . Por último, se redactarán de nuevo los guiones de prácticas para mejorar su claridad y se hará más hincapié en el tema de tratamiento de errores.

\section{AGRADECIMIENTOS}

P.E. Merino-Alonso agradece al Ministerio de Educación y Formación Profesional (MEyFP) por la beca FPU17/05433, que le permite desarrollar su tesis doctoral.

\section{REFERENCIAS}

Bloom, B. S. (1956). Taxonomy of educational objectives. Vol. 1: Cognitive domain. New York: McKay, 20, 24.

Gibson, G.E. et al. (2006) What is preproject planning, anyway? Journal of Management in Engineering, 22:3542. ASCE.

Godino-Gil, C. (1956). Teoría del buque y sus aplicaciones (Estática del buque). Barcelona: Gustavo-Gili S.A.

Pérez-Rojas, L. (1996). Los ensayos hidrodinámicos en la ingeniería naval, II Jornadas nacionales de Innovación en las enseñanzas de las Ingenierías, Área 2. Madrid, Instituto de Ciencias de la Educación. 